\title{
A Method for Discount with Maximum Profits in Fuzzy Environment Sense
}

\author{
N. Nalini, Research scholar, \\ Mathematics Department, \\ Presidency College/ University of Madras, India \\ Email:nrkhome@yahoo.com \\ Dr.E.Chandrasekaran \\ Professor \\ Department of Mathematics \\ Veltech Dr.RR \& Dr.SR University, India \\ e_chandrasekaran@yahoo.com
}

\begin{abstract}
To make padded set hypothesis and the delicateness in the stock issue, the desire is to discover the Revenue for defective total dissects to the aggregate cost and the related cost of offering estimation of updated thing correspondingly fluffy. Procedures/Statistical Analysis: This reasoning is to discover the aggregate which has the base cost with most unmistakable favorable position. Right when the favorable position got from offering one unit of the thing with affirmation periods and insurance period Up method cost and particularly planned Discounted costs must be gives some rate of focal points. Halfway favorable position of organized operation on stock clearing and powerful offering approachs, sensible benefit of the picking things must be finished. Exposures: Mathematical model has been made in underneath courses, (i) to locate the padded Up framework with the woolen material cost (ii) pay related with cushy period cost and preferred standpoint of the unmistakable bundle sizes of different Discounted things (iii)to locate the Fuzzy up technique with the fluffy screening expense of defective entirety with Advertisement subordinate pertinent cost tends to benefits with deficient misfortune (iv) extreme extra discounts for clearing and aggregate favorable position of the amazing number of stock even in the credit time traverse. Applications/Improvements: Our demonstrate is find completed cost and Profit with padded sense. Numerical frameworks are given and affectability examination is done to finish the outcome.
\end{abstract}

Keywords: Properties and Operators of fluffy Number, Up process, Relevant cost, Screening cost, Discount, Interest, Fuzzy Revenue, Fuzzy Profit, .

\section{INRODUCTION:}

The notice make various things, one side harmed things losing brand values, inverse side overhauled with diminished aftereffects of constrained time offers. Connection of scrap qualities to totally profitable Up process enlarge the advantage, that is Down trading move to Up Process. Diminished things are remanufactured oncegoverns with notice to the market with leader of pride due to acknowledgment. Unique offers and diminished expenses will attract the customers, non-clear Up process will do each one of the parts of Profit making instead of partial adversity in industry which will grow the wage. End we have to pay the excitement for acquiring aggregate under the trade credit conditions. 
Indicate cost consolidates holding cost, screening cost, refund cost, business cost and eagerness of obtainment cost. True blue screening and segregating methodology of stock will brings the pay more. Set apart down rates increase infers indicate squander thing level will reduces, in light of the fact that each one of the customers are pulled in by the discount not by scrap. Now and again, scraps will be used for models for educating and raining purposes like old plan engines, Ship parts, boundary vehicles, mobile phones, contraptions, electronic things, overpowering vehicle parts and planes parts, so scrap or total waste tends to informational models used for get ready purposes.

The Defective things with quick return broke down and found the arrangement of positioning technique with fuzzified buy cost and important cost with triangular fluffy number1. The trapezoidal fluffy request and extra request design with Shortages and multiplied condition2. Blemished things sold subsequent to screening procedure and profound on that, for the most part in exchange returns products goes to assembling as it were. Be that as it may, what is the utilization of that? Significant cost and sending the defectives are misuse of cash, however legitimate getting ready for defectives will make an extra benefit with halfway Loss3, Developed an exchange acknowledge display for multivariable request and the target of survey the system with multivariate request under lessening conditions has been solved4. Numerical model for Economic Order amount show with quick return of deficient things 5. A great EOQ show with settled values and buy, pertinent expenses are included with rebates and incomplete weakening costs6. Requesting, Holding expense and security stock with fluffy number-crunching Operators7.Wellknown monetary request amount show request with admissible deferral in installments with arrangements analysed8.Basic hypothesis of administrators and various part estimate and preplanned gainful return of goods9, 11. The incomplete accumulating stock model for decaying things considering stock and value delicate request rate in fluffy sense10. A multithing stock model of weakening things with close date is created and analyzed12. Late patterns and correlation of different creators in stock control strategy13.Determined the ideal generation cycle which has a tendency to diminish the aggregate cost of the stock system14. To boost the resultant Profit and it contain with request and reordering process15. Instructions to decrease cost of enlistment, commercial, holding, travel recompense and administration cost are discussed 16. Fractional multiplying and crumbling represents with differential equations17.Considering an ideal stock model for disintegrating things and Demand rate is depends cost and understood the model for aggregate profit18. Left and Right Triangular fluffy numbers used to discover important cost and return of merchandise cost19. Created two phase booking for to minimize rental cost under fluffy time utilizing triangular fluffy number can be stretched out to trapezoidal fluffy numbers with various parameters20. Basic Applications on operations on summed up trapezoidal fluffy numbers21. Operations on trapezoidal fluffy numbers with unverifiable conditions22.Defective thing arrangement depends with steady request and time developed23.The standard of assembling system is to got ideal fluffy rule24.25analysed fluffy trapezoidal govern for normal mean esteem method26. Fluffy enhancement assumes the principle part in information mining and share advertise tradings 27. 
The notice make various things, one side harmed things losing brand values, inverse side updated with decreased aftereffects of constrained time offers. Connection of scrap qualities to totally beneficial Up process expand the advantage, that is Down trading move to Up Process. Lessened things are remanufactured oncegoverns with notice to the market with leader of pride in light of acknowledgment. Exceptional offers and diminished expenses will attract the customers, nondirect Up process will do each one of the parts of Profit making instead of fragmentary setback in industry which will extend the wage. End we have to pay the eagerness for acquiring total under the trade credit conditions.

Indicate cost fuses holding cost, screening cost, refund cost, business cost and excitement of acquirement cost. True blue screening and secluding methodology of stock will brings the wage more. Set apart down rates increase infers mean waste thing level will decreases, in light of the fact that each one of the customers are pulled in by the refund not by scrap. At times, scraps will be used for models for educating and raining purposes like old plan engines, Ship parts, hindrance vehicles, mobile phones, contraptions, electronic things, overpowering vehicle parts and planes parts, so scrap or total waste tends to educational models used for get ready purposes.

\section{NOTATIONS AND ASSUMPTIONS:}

$\sim$ wavy bar indicates fuzzification of parameters.

$\widetilde{\mathrm{P}_{\mathrm{Uc}}}$ is the Purchase cost of items per unit.

$\widetilde{\mathrm{UP}}$ is the Up Process with defective items.

$\widetilde{\mathrm{D}_{\mathrm{q}}}$ is the Fuzzy Demand quantity

$\widetilde{\mathrm{H}}$ is the Holding cost

$\widetilde{0}$ is the Ordering or setup cost

$\tilde{r}$ is the Order quantity in Discounted Process

$\widetilde{L_{r}}$ is the Length of the plan in discounted period.

$\widetilde{L_{1}}$ is the Length of the plan in Discounted Process(30days)

$\widetilde{L_{2}}$ is the Length of the plan in Discounted process with interest payable period(>30days)

$\widetilde{L_{3}}$ is the Length of the plan inDiscounted processinterest payable process (>60days)

$\mathrm{R}_{1}$ is the Testing and dismantling (depreciation cost)

$\mathrm{R}_{2}$ is the Labor cost

$\mathrm{R}_{3}$ is the Additional Material cost/ Upgrading and quality test cost. 
$\mathrm{S}_{\mathrm{g}}$ is the segregation cost of a lot.

$\mathrm{T}_{\mathrm{w}}$ is the total waste in a lot.

$\mathrm{P}_{\mathrm{S}}$ is he proper screening of a unit.

$\mathrm{Q}_{\mathrm{c}}$ is the Quality Check cost of a unit.

$A_{d}$ is the Advertisement cost per unit time.

$\mathrm{I}_{\mathrm{p}}$ is the interest percentage of credit purchase of a period.

$\mathrm{TI}_{\mathrm{C}}$ is the Total Cost with Interest.

$\mathrm{I} \%$ is the interest rate

[ $\widetilde{\mathrm{TDS}}]$ is the Total Discounted Cost of a lot.

$\widetilde{S V}_{\mathrm{D}}$ is the Selling value of Defective items with discount per unit

$\widetilde{D S}_{p}$ is Discounted Percentage.

$\left[\mathrm{R}_{\mathrm{ev} 1} \mathrm{DS}\right]$ is the Revenue in Discouned items.

$\left[\widetilde{\mathrm{R}_{\mathrm{ev} 2} \mathrm{DS}}\right]$ is the Revenue in Discouned with credit situations.

$\left[\widetilde{\mathrm{P}_{1} \mathrm{DS}}\right]$ is the gain in Discount Process

$\left[\widetilde{\mathrm{P}_{2} \mathrm{DS}}\right]$ is the gain in Discount with credit situations.

\section{MATHEMATICAL MODEL FORMULATIONS:}

\subsection{To find the Holding cost ordering cost for discounted items,}

Holding/ Rental Cost in Fuzzy Sense $=\widetilde{\mathrm{H}} \otimes \widetilde{\mathrm{r}} \otimes \widetilde{\mathrm{L}}_{\mathrm{r}}$

Ordering cost in fuzzy sense $=\widetilde{0} \otimes \tilde{\mathrm{r}}$ (2)

Total Cost is addition of holding cost and Setup cost,

Primarily cost items which includes total cost,

\subsection{To find total cost,}

From equation (1), (2),

$$
\text { Total cost in fuzzy }=\left[\widetilde{\mathrm{P}_{\mathrm{Uc}}} \otimes \tilde{\mathrm{r}}\right] \oplus\left[\widetilde{\mathrm{H}} \otimes \widetilde{\mathrm{r}} \otimes \widetilde{\mathrm{L}}_{\mathrm{r}}\right] \oplus[\widetilde{\mathrm{O}} \otimes \tilde{\mathrm{r}}]
$$

$[\widetilde{T D S}]=\left[\widetilde{\mathrm{P}_{\mathrm{Uc}}} \otimes \tilde{\mathrm{r}}\right] \oplus\left[\widetilde{H} \otimes \widetilde{r} \otimes \widetilde{L}_{r} \oplus \widetilde{O} \otimes \tilde{r}\right]$ 
In addition to that, $\mathrm{R}_{1}, \mathrm{R}_{2}, \mathrm{R}_{3}$ are repairing steps of dismantling, labor and additional materials.

Planning Process costs are Segregation, Quality check and Advertisement costs.

$[\widetilde{T D S}]=\left[\widetilde{\mathrm{P}_{\mathrm{Uc}}} \otimes \tilde{\mathrm{r}}\right] \oplus\left[\widetilde{H} \otimes \tilde{r} \otimes \widetilde{L}_{r} \oplus \widetilde{O} \otimes \tilde{r}\right] \oplus\left(T_{w}\right) \otimes \tilde{r}$

$$
\left(T_{w}\right)=[\mathrm{R} 1+\mathrm{R} 2+\mathrm{R} 3] \otimes \tilde{r} \oplus\left(P_{s}\right) \otimes \tilde{r} \oplus\left(Q_{c}\right) \otimes \tilde{r} \oplus\left(A_{d}\right) \otimes \widetilde{L_{r}}
$$

\subsection{Relevant cost of discounted items,}

Applying equation (5) in(4), we get,

$[\widetilde{T D S}]=\left[\widetilde{\mathrm{P}_{\mathrm{Uc}}} \otimes \tilde{\mathrm{r}}\right] \oplus\left[\widetilde{H} \otimes \tilde{r} \otimes \widetilde{L}_{r} \oplus \widetilde{O} \otimes \tilde{r}\right] \oplus[\mathrm{R} 1+\mathrm{R} 2+\mathrm{R} 3] \otimes \tilde{r} \oplus\left(P_{s}\right) \otimes \tilde{r} \oplus$ $\left(Q_{c}\right) \otimes \tilde{r} \oplus\left(A_{d}\right) \otimes \widetilde{L_{r}}$

Here, Advertisement cost for one time which includes Television advertisement, internet, newspapers, notices and blockades etc.

\subsection{To find Revenue in Discounted situations,}

Let $\widetilde{D S}_{p}=\left(\widetilde{D S}_{1}, \widetilde{D S_{2}}, \widetilde{D S_{3}}, \widetilde{D S_{4}}, \widetilde{D S_{5}}, \widetilde{D S_{6}}, \widetilde{D S_{7}}, \widetilde{D S_{8}}, \widetilde{D S_{9}}\right)$ and $\widetilde{S V}_{D}=$

$\left(\widetilde{S V}_{1}, \widetilde{S V}_{2}, \widetilde{S V}_{3}, \widetilde{S V}_{4}, \widetilde{S V}_{5}, \widetilde{S V}_{6}, \widetilde{S V}_{7}, \widetilde{S V}_{8}, \widetilde{S V}_{9}\right)$ are Fuzzy numbers then,

$\left[\widetilde{R_{e v} D}\right.$ ]

$=$ Selling Price X Discounted percentage X Quantity of items in with discounted prices

$$
\begin{aligned}
{\left[\widetilde{R_{e v} D} s\right]=} & {\left[\widetilde{S V}_{D} \otimes \widetilde{D S}_{p} \otimes \tilde{r}\right] } \\
= & {\left[\left(\widetilde{S V}_{1}, \widetilde{S V}_{2},{\widetilde{S V_{3}}}^{S V_{4}}, \widetilde{S V}_{5}, \widetilde{S V}_{6}, \widetilde{S V}_{7}, \widetilde{S V}_{8}, \widetilde{S V}_{9}\right)\right.} \\
& \left.\otimes\left(\widetilde{D S_{1}}, \widetilde{D S_{2}}, \widetilde{D S_{3}}, \widetilde{D S_{4}}, \widetilde{D S_{5}}, \widetilde{D S_{6}}, \widetilde{D S_{7}}, \widetilde{D S_{8}}, \widetilde{D S_{9}}\right) \otimes \tilde{r}\right]
\end{aligned}
$$

$\left[\widetilde{R_{e v 1} D} s\right]$

$=\left[\widetilde{S V}_{1} \otimes \widetilde{D S}_{1} \otimes \tilde{r}, \widetilde{S V}_{2} \bigotimes \widetilde{D S}_{2}\right.$

$\otimes \tilde{r}, \widetilde{S V}_{3} \bigotimes \widetilde{D S}_{3} \otimes \tilde{r}, \widetilde{S V}_{4} \bigotimes \widetilde{D S}_{4} \otimes \tilde{r}, \widetilde{S V}_{5} \bigotimes \widetilde{D S}_{5} \otimes \tilde{r}, \widetilde{S V}_{6} \bigotimes \widetilde{D S}_{6} \otimes \tilde{r}, \widetilde{S V}_{7} \bigotimes \widetilde{D S}_{7} \otimes \tilde{r}$,

$\left.\widetilde{S V}_{8} \bigotimes \widetilde{D S}_{8} \otimes \tilde{r}, \widetilde{S V}_{9} \bigotimes \widetilde{D S}_{9} \otimes \tilde{r}\right]$

\subsection{To find the Profit in discounted situations,}


Profit $=$ Revenue - Total Cost

Using equations (6) and (7), we get,

$$
\left[\widetilde{P_{F} D S}\right]=\left[\widetilde{R_{e v} D s}\right] \ominus[\widetilde{T D S}]
$$

Here, $\mathrm{F}=(1,2)$ in different Profits in two stages.

$\left[\widetilde{P_{1} D s}\right]=$

$\left[\widetilde{S V}_{1} \otimes \widetilde{D S}_{1} \otimes \tilde{r}, \widetilde{S V}_{2} \otimes \widetilde{D S}_{2} \otimes\right.$

$r$, $S V 3 D S 3 \otimes r, S V 4 D S 4 \otimes r, S V 5 D S 5 \otimes r, S V 6 D S 6 \otimes r, S V 7 D S 7 \otimes r$, $S V 8 D S 8 \otimes r, S V 9 D S 9 \otimes r \ominus \mathrm{PUc} \quad \otimes \mathrm{r} \oplus H \otimes r \otimes L r \oplus O \otimes r \oplus \mathrm{R}_{1+} \mathrm{R}_{2+} \mathrm{R}_{3]} \otimes r \oplus(P S)$ $\otimes \tilde{r} \oplus\left(Q_{c}\right) \otimes \tilde{r} \oplus\left(A_{d}\right) \otimes \widetilde{L_{r}}\{$ by the equation (7) and (6)

$$
\begin{aligned}
& {\left[\widetilde{P_{1} D S}\right]=\left[\widetilde { S V _ { 1 } } \otimes \widetilde { D S _ { 1 } } \otimes \tilde { r } \ominus \left\{\left[\widetilde{\mathrm{P}_{\mathrm{Uc}}} \quad \otimes \tilde{r}\right] \oplus\left[\widetilde{H} \otimes \widetilde{r} \otimes \widetilde{L}_{r} \oplus \widetilde{O} \otimes \tilde{r}\right] \oplus[\mathrm{R} 1+\mathrm{R} 2+\mathrm{R} 3]\right.\right.} \\
& \left.\otimes \tilde{r} \oplus\left(P_{S}\right) \otimes \tilde{r} \oplus\left(Q_{c}\right) \otimes \tilde{r} \oplus\left(A_{d}\right) \otimes \widetilde{L_{r}}\right\}, \widetilde{S V_{2}} \bigotimes \widetilde{D S_{2}} \otimes \tilde{r} \ominus\left\{\left[\widetilde{\mathrm{P}_{\mathrm{Uc}}} \otimes \tilde{r}\right]\right. \\
& \oplus\left[\widetilde{H} \otimes \tilde{r} \otimes \widetilde{L}_{r} \oplus \widetilde{O} \otimes \tilde{r}\right] \oplus[\mathrm{R} 1+\mathrm{R} 2+\mathrm{R} 3] \otimes \tilde{r} \oplus\left(P_{s}\right) \otimes \tilde{r} \oplus\left(Q_{c}\right) \otimes \tilde{r} \\
& \left.\oplus\left(A_{d}\right) \otimes \widetilde{L_{r}}\right\}, \widetilde{S V}_{3} \bigotimes \widetilde{D S_{3}} \otimes \tilde{r} \ominus\left\{\left[\widetilde{\mathrm{P}_{\mathrm{Uc}}} \otimes \tilde{r}\right] \oplus\left[\widetilde{H} \otimes \widetilde{r} \otimes \widetilde{L}_{r} \oplus \widetilde{O} \otimes \tilde{r}\right]\right. \\
& \bigoplus[\mathrm{R} 1+\mathrm{R} 2+\mathrm{R} 3] \otimes \tilde{r} \oplus\left(P_{s}\right) \otimes \tilde{r} \oplus\left(Q_{c}\right) \otimes \tilde{r} \oplus\left(A_{d}\right) \\
& \left.\otimes \widetilde{L_{r}}\right\}, \widetilde{S V_{4}} \bigotimes \widetilde{D S_{4}} \otimes \tilde{r} \ominus\left\{\left[\widetilde{\mathrm{P}_{\mathrm{Uc}}} \otimes \tilde{r}\right][\mathrm{R} 1+\mathrm{R} 2+\mathrm{R} 3] \otimes \tilde{r} \oplus\left(P_{s}\right) \otimes \tilde{r}\right. \\
& \left.\oplus\left(Q_{c}\right) \otimes \tilde{r} \oplus\left(A_{d}\right) \otimes \widetilde{L_{r}}\right\}, \widetilde{S V}_{5} \otimes \widetilde{D S}_{5} \otimes \tilde{r} \ominus\left\{\left[\widetilde{\mathrm{P}_{\mathrm{Uc}}} \otimes \tilde{r}\right]\right. \\
& \oplus\left[\widetilde{H} \otimes \tilde{r} \otimes \widetilde{L}_{r} \oplus \widetilde{O} \otimes \tilde{r}\right] \oplus[\mathrm{R} 1+\mathrm{R} 2+\mathrm{R} 3] \otimes \tilde{r} \oplus\left(P_{s}\right) \otimes \tilde{r} \oplus\left(Q_{c}\right) \otimes \tilde{r} \\
& \left.\oplus\left(A_{d}\right) \otimes \widetilde{L_{r}}\right\}, \widetilde{S V_{6}} \otimes \widetilde{D S_{6}} \otimes \tilde{r} \ominus\left\{\left[\widetilde{\mathrm{P}_{\mathrm{Uc}}} \otimes \tilde{r}\right] \oplus\left[\widetilde{H} \otimes \tilde{r} \otimes \widetilde{L}_{r} \oplus \widetilde{O} \otimes \tilde{r}\right]\right. \\
& \oplus[\mathrm{R} 1+\mathrm{R} 2+\mathrm{R} 3] \otimes \tilde{r} \oplus\left(P_{s}\right) \otimes \tilde{r} \oplus\left(Q_{c}\right) \otimes \tilde{r} \oplus\left(A_{d}\right) \otimes \widetilde{L_{r}}, \widetilde{S V}_{7} \otimes \widetilde{D S_{7}} \otimes \tilde{r} \\
& \ominus\left\{\left[\widetilde{\mathrm{P}_{\mathrm{Uc}}} \otimes \tilde{r}\right] \oplus\left[\widetilde{H} \otimes \tilde{r} \otimes \widetilde{L}_{r} \oplus \widetilde{O} \otimes \tilde{r}\right] \oplus[\mathrm{R} 1+\mathrm{R} 2+\mathrm{R} 3] \otimes \tilde{r} \oplus\left(P_{s}\right)\right. \\
& \left.\otimes \tilde{r} \oplus\left(Q_{c}\right) \otimes \tilde{r} \oplus\left(A_{d}\right) \otimes \widetilde{L_{r}}\right\}, \widetilde{S V_{8}} \otimes \widetilde{D S_{8}} \otimes \tilde{r} \ominus\left\{\left[\widetilde{\mathrm{P}_{\mathrm{Uc}}} \otimes \tilde{r}\right]\right. \\
& \oplus\left[\widetilde{H} \otimes \tilde{r} \otimes \widetilde{L}_{r} \oplus \widetilde{O} \otimes \tilde{r}\right] \oplus[\mathrm{R} 1+\mathrm{R} 2+\mathrm{R} 3] \otimes \tilde{r} \oplus\left(P_{s}\right) \otimes \tilde{r} \oplus\left(Q_{c}\right) \otimes \tilde{r} \\
& \left.\oplus\left(A_{d}\right) \otimes \widetilde{L_{r}}\right\}, \widetilde{S V_{9}} \otimes \widetilde{D S_{9}} \otimes \tilde{r} \ominus\left\{\left[\widetilde{\mathrm{P}_{\mathrm{Uc}}} \otimes \tilde{r}\right] \oplus\left[\widetilde{H} \otimes \widetilde{r} \otimes \widetilde{L}_{r} \oplus \widetilde{O} \otimes \tilde{r}\right]\right. \\
& \left.\left.\oplus[\mathrm{R} 1+\mathrm{R} 2+\mathrm{R} 3] \otimes \tilde{r} \oplus\left(P_{s}\right) \otimes \tilde{r} \oplus\left(Q_{c}\right) \otimes \tilde{r} \oplus\left(A_{d}\right) \otimes \widetilde{L_{r}}\right\}\right]
\end{aligned}
$$

Screening cost and repairing cost includes labor salary allowances also. Quality check by high sensitive sensors and visible discernible, after 30 days, product is not cleared means distributor has to pay the interest of the products.

Discounted cost varies for different durations, stock lot clearing in short period, we can get revenue without any delay. More percentage discounts will attract the customers to tempting to buy. 
$\left[\widetilde{P_{1} D S}\right]=\left[\widetilde{S V_{1}} \otimes\left(1-\widetilde{\left.D S_{1}\right)} \otimes \tilde{r} \ominus\left\{\left[\widetilde{\mathrm{P}_{\mathrm{Uc}}} \quad \otimes \tilde{r}\right] \oplus\left[\widetilde{H} \otimes \widetilde{r} \otimes \widetilde{L}_{r} \oplus \widetilde{O} \otimes \tilde{r}\right] \oplus[\mathrm{R} 1+\mathrm{R} 2+\right.\right.\right.$ $\left.\mathrm{R}_{3} \otimes r \oplus P S \otimes r \oplus Q c \otimes r \oplus A d \otimes L r\right\}, S V 2 \quad(1-D S 2) \otimes r \ominus\{\mathrm{PUc} \quad \otimes r \oplus H \otimes r \quad \otimes L r \oplus O \otimes r$ $\left.\oplus \mathrm{R}_{1+} \mathrm{R}_{2+} \mathrm{R}_{3} \otimes r \oplus P S \otimes r \oplus Q c \otimes r \oplus A d \otimes L r\right\}, S V 3(1-D S 3) \otimes r \ominus\{\mathrm{PUc} \quad \otimes r \oplus H \otimes r \otimes L r \oplus O$ $\left.\otimes r \oplus \mathrm{R}_{1+} \mathrm{R}_{2+} \mathrm{R}_{3} \otimes r \oplus P s \otimes r \oplus Q c \otimes r \oplus A d \otimes L r\right\}, S V 4(1-D S 4) \otimes r \ominus\left\{\mathrm{PUc} \quad \otimes r \mathrm{R}_{1+} \mathrm{R}_{2+}\right.$ $\left.\mathrm{R}_{3} \otimes r \oplus P S \otimes r \oplus Q c \otimes r \oplus A d \otimes L r\right\}, S V 5 \otimes(1-D S 5) \otimes r \ominus\{\mathrm{PUc} \quad \otimes r \oplus H \otimes r \quad \otimes L r \oplus O \otimes r$ $\left.\oplus \mathrm{R}_{1+} \mathrm{R}_{2+} \mathrm{R}_{3} \otimes r \oplus P S \otimes r \oplus Q c \otimes r \oplus A d \otimes L r\right\}, S V 6 \otimes(1-D S 6) \otimes r \ominus\{\mathrm{PUc} \quad \otimes r \oplus H \otimes r \quad \otimes$ $\left.L r \oplus O \quad \otimes r \quad \oplus \mathrm{R}_{1+} \quad \mathrm{R}_{2+} \quad \mathrm{R}_{3} \otimes r \oplus P s \otimes r \oplus Q c \otimes r \oplus A d \otimes L r\right\}, S V 7 \otimes(1-D S 7) \otimes r \ominus\{\mathrm{PUc}$ $\otimes r \oplus H \otimes r \quad \otimes \quad L r \oplus O \quad \otimes r \quad \mathrm{R}_{1+} \quad \mathrm{R}_{2+}$ $\left.\mathrm{R}_{3} \otimes r \oplus P S \otimes r \oplus Q c \otimes r \oplus A d \otimes L r\right\}, S V 8 \otimes(1-D S 8) \otimes r \ominus\{\mathrm{PUc} \quad \otimes r \oplus H \otimes r \quad \otimes L r \oplus O \quad \otimes r$ $\left.\oplus \mathrm{R}_{1+} \mathrm{R}_{2+} \mathrm{R}_{3} \otimes r \oplus P S \otimes r \oplus Q c \otimes r \oplus A d \otimes L r\right\}, S V 9 \otimes(1-D S 9) \otimes r \ominus\{\mathrm{PUc} \quad \otimes r \oplus H \otimes r \quad \otimes$ $L r \oplus O \otimes r \oplus\left[\mathrm{R}_{1+} \mathrm{R}_{2+} \mathrm{R}_{3]} \otimes r \oplus\left(P_{S}\right) \otimes r \oplus(Q c) \otimes r \oplus(A d) \otimes L r\right\} \quad$ (8)

Here, $\mathrm{D}_{\mathrm{s}}$ and $\mathrm{T}_{\mathrm{w}}$ are the discount cost and total waste items inversely proportional to each other.

\subsection{Relevant cost of discounted items with interest,}

To find the Holding cost and no ordering cost discounted and trade credit items

Primarily cost items which includes total cost and interest for $\mathrm{L}_{\mathrm{r}}$ Period, $\left[\widetilde{\mathrm{P}_{2} \mathrm{Ds}}\right]=\left[\left[\widetilde{\mathrm{SV}}_{1} \otimes(1-\right.\right.$ $\mathrm{DS} 1) \otimes \mathrm{r} \ominus\left\{\mathrm{PUc} \quad \otimes \mathrm{r} \oplus \mathrm{H} \otimes \mathrm{r} \quad \otimes \mathrm{Lr} \oplus \mathrm{O} \otimes \mathrm{r} \oplus \mathrm{R}_{1+} \mathrm{R}_{2+} \mathrm{R}_{3} \otimes \mathrm{r} \oplus \mathrm{Ps} \otimes \mathrm{r} \oplus \mathrm{Q} \mathrm{c} \otimes \mathrm{r} \oplus \mathrm{Ad} \otimes \mathrm{Lr}\right.$ $\ominus \mathrm{PUc} \otimes \mathrm{Lr} \otimes \mathrm{I} \%\}, \mathrm{SV} 2 \quad \otimes(1-\mathrm{DS} 2) \otimes \mathrm{r} \ominus\left\{\mathrm{PUc} \quad \otimes \mathrm{r} \oplus \mathrm{H} \otimes \mathrm{r} \otimes \mathrm{Lr} \oplus \mathrm{O} \otimes \mathrm{r} \oplus \mathrm{R}_{1+} \mathrm{R}_{2+}\right.$ $\mathrm{R} 3 \otimes \mathrm{r} \oplus \mathrm{Ps} \otimes \mathrm{r} \oplus \mathrm{Qc} \otimes \mathrm{r} \oplus \mathrm{Ad} \otimes \mathrm{Lr} \ominus \mathrm{PUc} \otimes \mathrm{Lr} \otimes \mathrm{I} \%\}, \mathrm{SV} 3 \otimes(1-\mathrm{DS} 3) \otimes \mathrm{r} \ominus \mathrm{PUc} \quad \otimes \mathrm{r} \oplus \mathrm{H} \otimes \mathrm{r} \otimes$ $\mathrm{Lr} \oplus 0 \quad \otimes \mathrm{r} \quad \oplus \mathrm{R}_{1+} \quad \mathrm{R}_{2+} \quad \mathrm{R}_{3} \otimes \mathrm{r} \oplus \mathrm{Ps} \otimes \mathrm{r} \oplus \mathrm{Qc} \otimes \mathrm{r} \oplus \mathrm{Ad} \otimes \mathrm{Lr} \ominus \mathrm{PUc} \otimes \mathrm{Lr} \otimes \mathrm{I} \%, \quad \mathrm{SV} 4 \quad \otimes(1-$ DS4) $\otimes r \ominus\left\{P U c \quad \otimes \mathrm{rR}_{1+} \mathrm{R}_{2+} \mathrm{R}_{3} \otimes \mathrm{r} \oplus \mathrm{Ps} \otimes \mathrm{r} \oplus \mathrm{Qc} \otimes \mathrm{r} \oplus \mathrm{Ad} \otimes \mathrm{Lr} \ominus \mathrm{PUc} \otimes \mathrm{Lr} \otimes \mathrm{I} \%\right\}, \mathrm{SV} 5 \otimes(1-$ DS5) $\otimes \mathrm{r} \ominus\left\{\mathrm{PUc} \quad \otimes \mathrm{r} \oplus \mathrm{H} \otimes \mathrm{r} \quad \otimes \quad \mathrm{Lr} \oplus \mathrm{O} \quad \otimes \mathrm{r} \quad \mathrm{PR}_{1+} \quad \mathrm{R}_{2+}\right.$ $\left.\mathrm{R}_{3} \otimes \mathrm{r} \oplus \mathrm{Ps} \otimes \mathrm{r} \oplus \mathrm{Qc} \otimes \mathrm{r} \oplus \mathrm{Ad} \otimes \mathrm{Lr} \ominus \mathrm{PUc} \otimes \mathrm{Lr} \otimes \mathrm{I} \%\right\}, \mathrm{SV} 6 \otimes(1-\mathrm{DS} 6) \otimes \mathrm{r} \ominus\{\mathrm{PUc} \quad \otimes \mathrm{r} \oplus \mathrm{H} \otimes \mathrm{r} \quad \otimes$ $\mathrm{Lr} \oplus 0 \quad \otimes \mathrm{r} \quad \mathrm{R}_{1+} \quad \mathrm{R}_{2+}$ $\left.\mathrm{R}_{3} \otimes \mathrm{r} \oplus \mathrm{Ps} \otimes \mathrm{r} \oplus \mathrm{Qc} \otimes \mathrm{r} \oplus \mathrm{Ad} \otimes \mathrm{Lr} \ominus \mathrm{PUc} \otimes \mathrm{Lr} \otimes \mathrm{I} \%\right\}, \mathrm{SV} 7 \otimes(1-\mathrm{DS} 7) \otimes \mathrm{r} \ominus\{\mathrm{PUc} \quad \otimes \mathrm{r} \oplus \mathrm{H} \otimes \mathrm{r} \quad \otimes$ $\mathrm{Lr} \oplus 0 \quad \otimes \mathrm{r} \quad \mathrm{R}_{1+} \quad \mathrm{R}_{2+}$ $\left.\mathrm{R}_{3} \otimes \mathrm{r} \oplus \mathrm{Ps} \otimes \mathrm{r} \oplus \mathrm{Qc} \otimes \mathrm{r} \oplus \mathrm{Ad} \otimes \mathrm{Lr} \ominus \mathrm{PUc} \otimes \mathrm{Lr} \otimes \mathrm{I} \%\right\}, \mathrm{SV} 8 \otimes(1-\mathrm{DS} 8) \otimes \mathrm{r} \ominus\{\mathrm{PUc} \quad \otimes \mathrm{r} \oplus \mathrm{H} \otimes \mathrm{r} \otimes$ $\mathrm{Lr} \oplus 0 \quad \otimes \mathrm{r} \quad \oplus \mathrm{R}_{1+} \quad \mathrm{R}_{2+}$ $\left.\mathrm{R}_{3} \otimes \mathrm{r} \oplus \mathrm{Ps} \otimes \mathrm{r} \oplus \mathrm{Qc} \otimes \mathrm{r} \oplus \mathrm{Ad} \otimes \mathrm{Lr} \ominus \mathrm{PUc} \otimes \mathrm{Lr} \otimes \mathrm{I} \%\right\}, \mathrm{SV} 9 \otimes(1-\mathrm{DS} 9) \otimes \mathrm{r} \ominus\{\mathrm{PUc} \quad \otimes \mathrm{r} \oplus \mathrm{H} \otimes \mathrm{r} \quad \otimes$ $\mathrm{Lr} \oplus 0 \otimes \mathrm{r} \oplus\left[\mathrm{R}_{1+} \mathrm{R}_{2+} \mathrm{R}_{3]} \otimes \mathrm{r} \oplus(\mathrm{Ps}) \otimes \mathrm{r} \oplus(\mathrm{Qc}) \otimes \mathrm{r} \oplus(\mathrm{Ad}) \otimes \mathrm{Lr} \ominus \mathrm{PUc} \otimes \mathrm{Lr} \otimes \mathrm{I} \%\right\}$

\section{Problem Calculations:}


Fabricating Defective merchandise in the Market with Repairable, Resalable condition things available to be purchased, Purchase cost of a Gadget is 1,000 Rupees for the Distributor under half Discount of Retail offering Price. One parcel size is 100 things, Rentals for keeping the Products is Rs.1/thing, requesting expense is Rs.1/thing, Repairing and Dismantling, Labor, Additional Material cost is $0.5 \%, 1 \%, 0.25 \%$ individually from the offering values. Culminate screening, Quality check, Advertisement cost are 0.05\%,0.08\%,0.12\% individually, Selling Percentage markdown and twofold rebates are in rates of $10 \%-90 \%$. Intrigue rate for buying expense is $10 \%$ for more than 30days up to 60 days, days may surpassing means loan fee may duplicates for each 30days.To increment the deal in intrigue payable period deals group will expand their notice expenses and deals related action cost are in twofold.

\subsection{Total Relevant Cost Table: 1}

\begin{tabular}{|c|c|c|c|l|l|l|l|l|l|l|c|}
\hline$\tilde{r}$ & $\widetilde{\mathrm{P}_{\mathrm{Uc}}}$ & $\widetilde{\mathrm{H}}$ & $\widetilde{\mathrm{O}}$ & $\mathrm{R}_{1}$ & $\mathrm{R}_{2}$ & $\mathrm{R}_{3}$ & $\mathrm{~A}_{\mathrm{d}}$ & $\mathrm{P}_{\mathrm{s}}$ & $\mathrm{Q}_{\mathrm{c}}$ & $\mathrm{A}_{\mathrm{d}}$ & {$[\widetilde{\mathrm{TDS}}]$} \\
\hline $\mathbf{1 0 0}$ & $\mathbf{1 0 0 0 0 0}$ & $\mathbf{3 0 0 0}$ & $\mathbf{1 0 0}$ & $\mathbf{5 0 0 0}$ & $\mathbf{6 0 0 0}$ & $\mathbf{2 5 0 0}$ & $\mathbf{1 2 0 0}$ & $\mathbf{5 0 0}$ & $\mathbf{8 0 0}$ & $\mathbf{1 2 0 0}$ & $\mathbf{1 2 0 3 0 0}$ \\
\hline
\end{tabular}

5.2. Interest Payable Table: 3

\begin{tabular}{|c|l|l|l|}
\hline \multicolumn{1}{|c|}{$\widetilde{\mathrm{P}_{\mathrm{Uc}}}$} & $\mathbf{I \%}$ & $\mathbf{L}_{\mathbf{r}}$ ( Period) & $\mathbf{T I}_{\mathbf{C}}$ \\
\hline 100000 & $(\mathbf{0 . 2 5}, \mathbf{0 . 5})$ & 30 & $(\mathbf{7 5 0 0 , 1 5 0 0 0 )}$ \\
\hline
\end{tabular}

5.2.1. Discount with and without interest Table:3:

\begin{tabular}{|c|c|c|c|c|c|}
\hline \multirow[t]{2}{*}{$\widetilde{D S}_{p}$} & \multirow[t]{2}{*}{$S \tilde{V}_{D}$} & \multirow[t]{2}{*}{$\widetilde{R_{e v 1} D S}$} & {$\left[\overline{\mathrm{P}_{1} \mathrm{DS}}\right]$} & {$\left[\widehat{\mathrm{P}_{2} \mathrm{DS}}\right]$} & {$\left[\widetilde{\mathrm{P}_{3} \mathrm{DS}}\right]$} \\
\hline & & & $\mathbf{I}=\mathbf{0}$ & $I=0.25$ & $I=0.5$ \\
\hline $4 \%$ & 1920 & 192000 & 71700 & 64200 & $\begin{array}{l}56700 \\
\end{array}$ \\
\hline $8 \%$ & 1840 & 184000 & 63700 & 56200 & 48700 \\
\hline $12 \%$ & 1760 & 176000 & 55700 & 48200 & 40700 \\
\hline $16 \%$ & 1680 & 168000 & 47700 & 40200 & 32700 \\
\hline $20 \%$ & 1600 & 160000 & 39700 & 32200 & 24700 \\
\hline $24 \%$ & 1440 & 144000 & 23700 & 16200 & 8700 \\
\hline $28 \%$ & 1360 & 136000 & 15700 & 8200 & 700 \\
\hline $32 \%$ & 1280 & 128000 & 7700 & 200 & -7300 \\
\hline $36 \%$ & 1200 & 120000 & 300 & -7200 & -14700 \\
\hline
\end{tabular}


No part payment allowed of ordered goods from the distributor to the manufacturer in the middle of the trade. At the same time rental for the keeping the place is same, we should give the rentals for the entire trade period.

\subsection{Graphical Representation of Discount verses Interest}

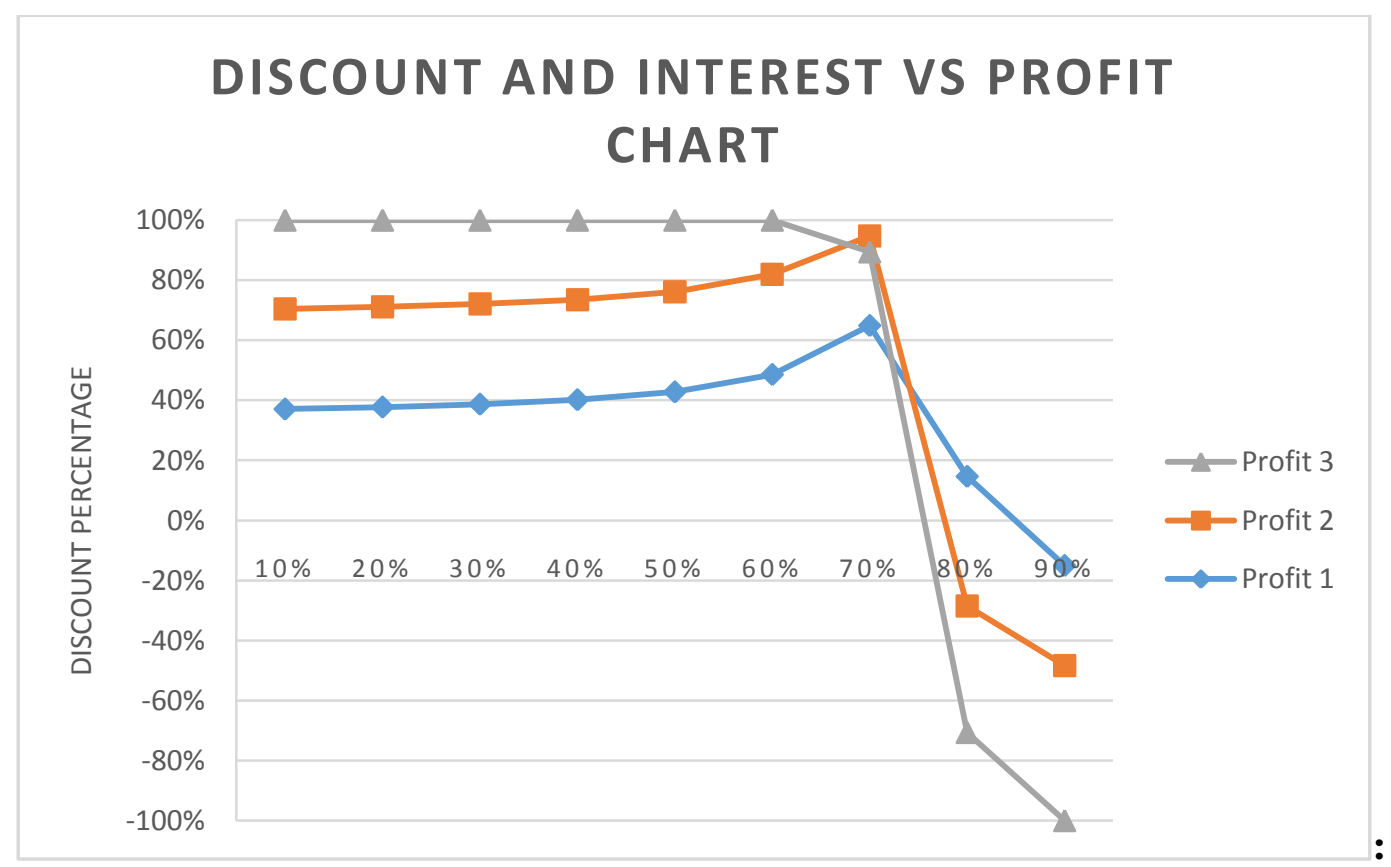

6. Conclusion:

Loss of cash happens in light of lacking things now and again achieves expected fragmentary hardship while others considered full difficulty. Point of fact, even consequently credit conditions also gives the best preferred standpoint with payable interest. In light of current conditions, if all purchasers are sitting tight at the Discount offering cost or go the going with thing. Specifically conditions a few clients will be able to sit tight for the going with requesting to fulfill their sales amidst the Discount day and age (or)Double Discount period episode can be dodged with Up strategy and advancement to that Discounted pay in two phases are filled the opening of the extension rate Moreover, graphical representations demonstrates that aggregate misfortune reaches to deficient preferred standpoint with Discounted and intrigue payable conditions.

From the tables (1) (2) and (3), it can be watched that,

(i) Revenue is decreases when in the refund Process.

(ii) Profit is Decreases when in the interest payable period.

(iii) Profit is immediate when in the Discount technique. 
(iv) Selling Price is lessening when in markdown get ready.

(v) Selling expense is exceptionally decreases in interest payable period.

(vi) Profit is consistent when in the refund system as opposed to mishap.

(vii) Partial Loss is addition when in high markdown handle instead of full setback.

(viii)Ordering and holding costs are same in all tables with respect to part gauge however Profit rate is contrasts due to refunds and eagerness losing the offering expense and brand regard in all over the place.

A Fuzzy stock model for demand with steady offering esteem, asking for cost and holding cost has been delivered with fleecy sense. Trapezoidal cushioned models are discovered income driven/Loss. A numerical case is moreover given in support the theory. A future research is to expand the model under unverifiable demand with different general incomes.

\section{References:}

1. Wen.kai and Kevin Hsu, "Ideal stock model with fluffy perfective rate, request rate and buying cost under prompt return for imperfect things", universal diary of imaginative figuring data and control,8(4) 2012,2583-2598

2. Yogendra Kumar Rajoria, S. R. Singh, Seema Saini ,"An Inventory Model for Decaying Item with Ramp Demand design under Inflation and Partial Backlogging",Indian Journal of Science and Technology,2015 June, 8(12), Doi no: 10.17485/ijst/2015/v8i12/52341

3. W.K. Hsu and H.F. yu,"Economic amount arrange demonstrate with prompt return for imperfect things", International diary of Innovative processing data and control",(5)2011,2215-2020.

4. Vashisth Vikram, Tomar Ajay, Shekhar Chandra, A. K. Malik,"A Trade Credit Inventory Model with Multivariate Demand for Non-Instantaneous Decaying products",Indian Journal of Science and Technology,2016 Apr, 9(15), Doi no:10.17485/ijst/2016/v9i15/84213

5. M.K. Salameh and M.Y. Jaber,"Economic arrange amount demonstrate for defective quality", global diary of generation and financial aspects, (64)2000,59-64.

6. Mohammad Reza Shahraki, Alireza Shahraki, Navid Javdan,"Developing an Inventory Mathematical Model with Deterioration Variables for Discounted Stochastic Goods",Indian Journal of Science and Technology,2014 Jan, 7(11), Doi no:10.17485/ijst/2014/v7i11/50447 
7. Chih-Hsun Hsieh,"optimization of fluffy stock models under fluffy request and fluffy lead time",Tamsui oxford diary of administration sciences,20(10)2004, 21-36.

8. Mukesh Bhagoria, C. M. Sadiwala, V. K. Khare,"Multilevel Inventory Techniques for Minimizing Cost-a Case Study", Indian Journal of Science and Technology,2010 June, 3(6), Doi no: 10.17485/ijst/2010/v3i6/29786

9. D. Dubois and H.Prade, Fuzzy sets and frameworks; Theory and Applications, Academic press, Newyork, 71-73.

10. Sanjay Sharma, S. R. Singh,"An Inventory Model for Decaying Items, considering Multi Variate Consumption Rate with Partial Backlogging",Indian Journal of Science and Technology,2013 July, 6(7), Doi no:10.17485/ijst/2013/v6i7/34338

11. Sommer. G, Fuzzy stock booking Applied frameworks and robotics, Newyork(6),1981,3052-3060.

12. Shilpy Tayal, S. R. Singhand Rajendra Sharma,"A Multi Item Inventory Model for Deteriorating Items with Expiration Date and Allowable Shortages",Indian Journal of Science and Technology, Vol 7(4), 463-471, April 2014.Doi no: $10.17485 / \mathrm{ijst} / 2014 / \mathrm{v} 7 \mathrm{i} 4 / 48636$.

13. Serhii Zuikov, "A writing audit on models of stock administration under instability, Business frameworks and Economics,5(1),2015.

14. Deo DattaAaryaand Mukesh Kumar, "Store network Model with Ramp Type Demand under Planning Horizon",Indian Journal of Science and Technology,8(15),DOI: 10.17485/ijst/2015/v8i15/51359, July 2015.

15. A.R. Roy, P. Dutta, D. Chakraborty,"An stock model for single period items with reordering openings under fluffy request", A global Journal of Computers and Mathematics with Applications, 53,2007, Elsevier, 1502-1517.

16. V. Nalina, M. Jeeva,"K-Release Inventory Model in Manpower Planning", ,Indian Journal of Science and Technology,2010 July, 3(7), Doi no: 10.17485/ijst/2010/v3i7/29803

17. D. Dutta, Pavan kumar, " A halfway multiplying stock model for decaying things with time shifting interest and holding cost", Croatian Operational Research survey, 2015, 321-334.

18.Rekha Rani Chaudhary and Vikas Sharma,"A Model for Weibull Deteriorate Items with Price Dependent Demand Rate and Inflation", Indian Journal of Science and Technology, Vol 8(10), 975-981, May 2015, DOI: 10.17485/ijst/2015/v8i10/51936 
19. Nirmal kumar Mandl,"Fuzzy EOQ display with positioning fluffy number cost parameters," Yugaslav Journal of Operations Research, 22,2012,247-264.

20. Kewal Krishan Nailwal, Deepak Gupta and Sameer Sharma, "Two Stage Flow Shop Scheduling under Fuzzy Environment",Indian Journal of Science and Technology, Vol 8(16), DOI: 10.17485/ijst/2015/v8i16/55529, July 2015.

21. Sanhita Banerjee,Tapan Kumar Roy," Arithmetic Operations on summed up Trapezoidal fluffy Number and its Applications", Turkish Journal of Fuzzy systems,(3)2012, 16-44.

22. A. Nagoor gani, S.N. Mohamed Assarudeen,"A new Operation on Triangular fluffy Number for taking care of fluffy straight programming issue", Applied Mathematical Sciences, 6,2012, 525-532.

23. Neha Mishra, Jitendra Kumar Soni," An EOQ stock model with fluffy crumbling rate and limited Production rate", IOSR diary of Mathematics,12(4),2012,1-9.

24. S. Manimaran, M. Ananthanarayanan,"A Study on Comparison between Fuzzy Assignment Problems Using Trapezoidal Fuzzy Numbers with Average Method", Indian Journal of Science and Technology,2012 Apr, 5(4), Doi no:10.17485/ijst/2012/v5i4/30432

25. Faramarz Karamizadeh, Seyed Ahad Zolfagharifar,"Using the Clustering Algorithms and Rule-based of Data Mining to Identify Affecting Factors in the Profit and Loss of Third Party Insurance, Insurance Company Auto",Indian Journal of Science and Technology,2016 Feb, 9(7), Doi no: 10.17485/ijst/2016/v9i7/87846

26.Farzaneh Heidarpoor, Farhad Sheikhi Shahrivar,"Unsystematic Risk and Internal Control Quality Impact on the Earning Quality by utilizing Volatility Profits Index as a part of Tehran Stock Exchange", Indian Journal of Science and Technology,2015 June, 8(11), Doi no:10.17485/ijst/2015/v8i11/71800.

27. N. Nalini, Dr. E. Chandrasekaran "An Inventory Model for Simple method for Profit finding with fluffy sense", International Journal of Innovative Research in Applied Sciences and Designing", Vol.1, Issue 1, pp.1-5. 